\title{
ON A LIPSCHITZ INVARIANT OF NORMED SPACES
}

\author{
YOAV BENYAMINI
}

(Communicated by William J. Davis)

\begin{abstract}
C. Bessaga introduced an invariant $\eta(X)$ for $\sigma$-compact normed linear spaces. He showed that $\eta(X)=\eta(Y)$ whenever $X$ and $Y$ are Lipschitz homeomorphic. In this note we construct two $\sigma$-compact normed spaces with $\eta(X)=\eta(Y)$ which are not Lipschitz homeomorphic. Moreover, there are no compact convex sets $K$ and $L$ generating $X$ and $Y$, respectively, which are Lipschitz homeomorphic. This answers two problems posed by Bessaga.
\end{abstract}

Let $K$ be a compact metric space. Denote by $e_{n}(K)$ the infimum of those $\varepsilon>0$ so that $K$ contains an $\varepsilon$-net of size $2^{n}$, and let $e(K)$ be the set of all positive sequences $\left(a_{n}\right)$ so that $\lim a_{n} / e_{n}(K)=0$. If $X$ is a $\sigma$-comapct metric space, put $\eta(X)=\cap\left(\cup_{n} e\left(A_{n}\right)\right)$, where the intersection is taken over all countable compact covers $\left(A_{n}\right)$ of $X$.

These definitions were introduced by C. Bessaga in [Bes], and used by him to construct a normed linear space $X$ which is not Lipschitz homeomorphic to its closed subspaces of codimension 1 . He showed that if $X$ is a closed subset of $Y$, or is a Lipschitz image of $Y$, then $\eta(X) \subseteq \eta(Y)$. In particular it follows that $\eta(X)=\eta(Y)$ when $X$ and $Y$ are Lipschitz homeomorphic.

At the end of his article, Bessaga asks if one can construct two non-Lipschitzhomeomorphic spaces for which $\eta(X)=\eta(Y)$ (Problem A), and whether $\eta(X)=\eta(Y)$ implies that there are Lipschitz-homeomorphic compact and convex sets $K$ and $L$ generating $X$ and $Y$, respectively (i.e., $X=\cup n K$ and $Y=\cup n L$ ) (Problem B).

In this short article we answer these problems by constructing $\sigma$-comapct normed linear spaces with $\eta(X)=\eta(Y)$, so that any compact convex set that generates $X$ cannot be Lipschitz homeomorphic to a subset of $Y$. Both the construction and the proofs are simple, and we use known techniques for reducing problems of Lipschitz classification to the linear case by differentiation.

We shall use standard notation and terminology (see for example [LT] and the survey article [Ben].

Let $\left\{p_{i}:-\infty<i<\infty\right\}$ and $\left\{q_{i}:-\infty<i<\infty\right\}$ be two doubly infinite

Received by the editors July 17, 1989.

1980 Mathematics Subject Classification (1985 Revision). Primary 46B20, 47H99.

This research was supported by the Fund for the Promotion of Research at the Technion and by the Technion V.P.R. fund-K. \& M. Bank Mathematics Research Fund. 
sequences so that $3<p_{i}<q_{i}<p_{i+1}<4$ for all $i$, and let

$$
Z_{p}=\left(\sum_{i} \oplus \ell_{p_{i}}\right)_{2}=\left\{x_{i j}:\left\|x_{i j}\right\|=\left(\sum_{i}\left(\sum_{j}\left|x_{i j}\right|^{p_{i}}\right)^{2 / p_{i}}\right)^{1 / 2}<\infty\right\} .
$$

Define similarly $Z_{q}=\left(\sum_{i} \oplus \ell_{q_{i}}\right)_{2}$, and put $K=\left\{x_{i j}:\left|x_{i j}\right| \leq 2^{-|i|-j}\right\}$.

$K$ is a compact and convex set when regarded as a subset of either $Z_{p}$ or $Z_{q}$, and we let $X_{p}$ be its linear span, $X_{p}=\cup n K$, with the $Z_{p}$ norm, and $X_{q}$ is the same linear space with the $Z_{q}$ norm.

$X_{p}$ and $X_{q}$ are $\sigma$-compact, and $\eta\left(X_{p}\right)=\eta\left(X_{q}\right)$, because each of these spaces is a Lipschitz image of the other. Indeed, as $p_{i}<q_{i}$ for all $i$, the formal identity is a norm - 1 linear operator from $X_{p}$ into $X_{q}$ which is obviously onto. Conversely, the map $T\left(x_{i j}\right)=\left(x_{i-1, j}\right)$ is a norm -1 operator from $X_{q}$ into $X_{p}$ (because $q_{i}<p_{i+1}$ for all $i$ ). It is also an onto map because $K / 2 \subseteq T(K) \subseteq 2 K$.

Proposition. Let $L$ be a compact convex subset of $X_{p}$ which generates $X_{p}$. Then $L$ cannot be Lipschitz homeomorphic to a subset of $Z_{q}$.

To prove the Proposition, we first need a few preliminaries. The first Lemma was used in [Bes, proof of Proposition 2]. We give the proof for the sake of completeness.

Lemma 1. Let $L$ be a compact convex subset of the normed space $X$ such that $X=\cup n L$, and let $K$ be a compact convex subset of $X$. Then there is an $n_{0}$ such that $K \subseteq n_{0} L$.

Proof. By the Baire Category Theorem, there are $n_{1}, x_{0} \in K$, and $\varepsilon>0$, so that $K \cap B\left(x_{0}, \varepsilon\right) \subseteq n_{1} L$. By the compactness and convexity of $K$, there is a $\delta>0$ such that $(1-\delta) x_{0}+\delta k \in K \cap B\left(x_{0}, \varepsilon\right) \subseteq n_{1} L$ for all $k \in K$; i.e., $K \subseteq \delta^{-1}\left(n_{1} L+(\delta-1) x_{0}\right) \subseteq n_{0} L$, provided $n_{0}$ is large enough, by the convexity of $L$.

Let $K$ be a subset of a Banach space $X$, and let $f$ be a function from $X$ into another Banach space $Y$. Fix a point $k$ in $K$, and let $X_{k}$ be the subspace of $X$ of all points $x$ such that $k+\lambda x \in K$ for small enough $|\lambda|$. The differential of $f$ at the point $k$, is a linear operator $D f_{k}: X_{k} \rightarrow Y$ such that $D f_{k}(x)=\lim _{\lambda \rightarrow 0}(f(k+\lambda x)-f(k)) / \lambda$ for all $x \in X_{k}$.

Note that if $f$ satisfies a Lipschitz condition with constant $C$, then $D f_{k}$ (when it exists) is a bounded linear operator of norm at most $C$. Similarly, if $f$ is a Lipschitz embedding, then $D f_{k}$ is an into linear isomorphism. Finally, if $X_{k}$ is dense in $X$ and $f$ is a Lipschitz embedding, then $D f_{k}$ extends by continuity to a linear isomorphism of all of $X$ into $Y$.

Our next Lemma is a summary of the results of $[\mathrm{M}, \S 4]$ in the context of Banach spaces, using a somewhat different terminology.

Lemma 2. Let $f$ be a Lipschitz function from a compact convex subset $K$ of a normed space $X$ into a Banach space $Y$ with the Radon-Nikodym Property. 
Assume $K$ is affinely homeomorphic to the Hilbert cube $Q$, and let $\mu$ be the image on $K$ of the usual measure on $Q$. Then $f$ has a differential $\mu$-a.e. on $K$.

The last lemma follows by a routine gliding-hump argument form the fact that $l_{r}$ is not isomorphic to a subspace of $l_{s}$ when $r \neq s$ (see [LT]).

Lemma 3. $Z_{p}$ is not linearly isomorphic to a subspace of $Z_{q}$.

Proof of proposition. By Lemma $1, L$ contains a multiple of $K$. It thus suffices to prove that $K$, considered as a subset of $Z_{p}$, cannot be Lipschitz-embedded into $Z_{q}$. But $K$ is affinely homeomorphic to the Hilbert cube and, for almost all points $k$ in $K, X_{k}$ is dense in $Z_{p}$. Also $Z_{q}$, being reflexive, has the Radon-Nikodym Property. It thus follows from Lemma 2 and the remarks preceding it that if $K$ were Lipschitz homeomorphic to a subset of $Z_{q}$, then $Z_{p}$ would be linearly isomorphic to a subspace of $Z_{q}$-but this contradicts Lemma 3 !

\section{REFERENCES}

[Ben] Y. Benyamini, The uniform classification of Banach spaces, Longhorn Notes, 1984-1985, pp. 15-38.

[Bes] C. Bessaga, A Lipschitz invariant of normed linear spaces related to the entropy numbers, Rocky Mountain J. Math. 10 (1980), 81-84.

[LT] J. Lindenstrauss and L. Tzafriri, Classical Banach spaces, Lecture Notes in Math., vol. 338, Springer-Verlag, New York, 1973.

[M] P. Mankiewicz, On the differentiability of Lipschitz mappings in Frechet spaces, Studia Math. 45 (1973), 15-29.

Department of Mathematics, Technion-Israel Institute of Technology, Haifa 32000 , ISRAEL 